\title{
Rendimiento clínico y uso de recursos en el arranque de una totalmente nueva unidad de cuidados intensivos. Mismo personal, nueva área
}

\author{
Clinical performance and resource utilization in the start of a totally new Intensive Care \\ Unit. Same staff, new area
}

\begin{abstract}
Pablo Álvarez-Maldonado"* Grisel Hernández-Ríos', Ulises Cerón-Díaz², Carlos Núñez-Pérez-Redondo, Francisco Navarro-Reynoso ${ }^{1}$ y Raúl Cicero-Sabido ${ }^{1,3}$

${ }^{1}$ Servicio de Neumología y Cirugía de Tórax, Hospital General de México Dr. Eduardo Liceaga; ${ }^{2}$ Unidad de Terapia Intensiva, Hospital Español de México; ${ }^{3}$ Facultad de Medicina, Universidad Nacional Autónoma de México. Ciudad de México, México
\end{abstract}

\section{Resumen}

Antecedentes: Existe poca información acerca del desempeño de una unidad de cuidados intensivos (UCI) cuando es reubicada en un área totalmente nueva y equipada. Objetivo: Analizar el rendimiento clínico y el uso de recursos de la nueva UCI respiratoria (UCIR) de un hospital grande de tercer nivel. Método: Estudio transversal, comparativo, con datos prospectivos de pacientes ingresados del 17 de julio de 2017 al 17 de julio de 2018. Se usa el método ajustado de Rapoport para obtener el índice de rendimiento clínico estandarizado (IRCE) y el índice de uso de recursos estandarizado (IRURE). Resultados: De 354 pacientes fueron excluidos los reingresos, los pacientes aún hospitalizados y aquellos a quienes se limitó o retiró el tratamiento. En 301 pacientes la sobrevida hospitalaria fue del 63\%, mientras que la sobrevida esperada fue del $67.7 \%$. EI IRCE fue -1.03 y el IRURE fue 0.05, situando el resultado en coordenadas dentro de dos desviaciones estándar en el gráfico de Rapoport. Hubo una diferencia estadísticamente significativa en la sobrevida comparando el periodo de estudio con resultados de la UCIR obtenidos antes de su reubicación (63 vs. 55\%, $p=0.01$ ). Conclusiones: En su primer año de funcionamiento, la nueva UCIR tuvo mejor rendimiento clínico que la antigua, sin modificación en el uso de recursos.

Palabras Clave: Rendimiento clínico. Uso de recursos. Unidad de cuidados intensivos.

\begin{abstract}
Background: There is little information of intensive care unit (ICU) performance when it's relocated to a totally new and equipped area. Objective: To analyze the clinical performance and use of resources of a new respiratory-ICU (nRICU) in a large third-level care hospital. Method: Cross-sectional, comparative study using prospective data of patients admitted from July 17, 2017 to July 17, 2018. The Rapoport adjusted method was used to obtain the standardized clinical performance index (SCPI) and the standardized resource use index (SRUI). Results: Out of 354 patients, those who were readmissions or remained hospitalized and those whose treatment was withheld or withdrawn where excluded from the analysis. In 301 patients, the observed survival at hospital discharge was $63 \%$ while the expected survival was $67.7 \%$. Values of SCPI and SRUI were -1.03 and 0.05 respectively, placing results in coordinates within two standard deviations when plotted in the Rapoport chart.
\end{abstract}

\section{Correspondencia:}

*Pablo Álvarez-Maldonado

Dr. Balmis, 148

Col. Doctores

Fecha de recepción: 26-06-2019

C.P. 06725, Ciudad de México, México

E-mail: pamyacs@yahoo.com
Cir Cir. 2020;88(3):337-343

Contents available at PubMed www.cirugiaycirujanos.com 0009-7411/@ 2019 Academia Mexicana de Cirugía. Publicado por Permanyer. Este es un artículo open access bajo la licencia CC BY-NC-ND (http://creativecommons.org/licenses/by-nc-nd/4.0/). 
Cirugía y Cirujanos. 2020;88(3)

There was a statistically significant difference in survival when comparing the study period with outcomes obtained in the RICU before its relocation ( $63 \%$ vs. $55 \%, p=0.01)$. Conclusions: In its 1 st year of operation, the $n R I C U$ had better clinical performance compared to the former RICU, with no change in the use of resources.

Key Words: Clinical performance. Use of resources. Intensive care unit.

\section{Introducción}

Dentro de los esfuerzos continuos que se desarrollan para mejorar el rendimiento de la unidad de cuidados intensivos (UCl), disponer de recursos y de un área adecuada para la atención de pacientes graves puede considerarse el punto de partida. Los análisis multicéntricos sostienen que un gasto excesivo en la $\mathrm{UCl}$ no necesariamente se asocia a un rendimiento clínico excepcional'; no obstante, se recomienda abordar factores específicos del entorno para mejorar el rendimiento de la $\mathrm{UCl}$ de modo que impacte en los pacientes ${ }^{2,3}$. Poco se sabe de los cambios en el rendimiento de una $\mathrm{UCl}$ cuando es reubicada a un área totalmente nueva con el mismo personal que la compone. Dotada de una construcción que data de inicios de los años 1900 y de equipamiento en su mayor parte no acorde con los estándares actuales, la unidad de cuidados intensivos respiratorios (UCIR) del Hospital General de México Dr. Eduardo Liceaga (el primer hospital de especialidades del país) dejó su localización en julio de 2017 para ser reubicada, junto con el servicio de neumología y cirugía de tórax, en un edificio completamente nuevo y equipado dentro del hospital. El presente estudio analiza el rendimiento clínico y el uso de recursos de la nueva UCIR durante su primer año de funcionamiento, y compara los resultados con los obtenidos antes de su reubicación.

\section{Método}

\section{Diseño}

Estudio transversal, comparativo y retrospectivo con datos obtenidos de manera prospectiva.

\section{Pacientes y lugar}

Se incluyen datos de pacientes que ingresaron del 17 de julio de 2017 al 17 de julio de 2018 a la nueva UCIR del Hospital General de México Dr. Eduardo Liceaga, un hospital público escuela de 1,131 camas. La nueva UCIR es una de las ocho unidades de terapia intensiva dentro del hospital, cuenta con 12 camas completamente equipadas y la compone un equipo multidisciplinario formado por cuatro médicos intensivistas certificados (repartidos en los turnos matutino, vespertino, noches $A$ [lunes, miércoles y viernes] y fines de semana [8 am a $8 \mathrm{pm}]$ ), cuatro médicos neumólogos certificados repartidos en diferentes turnos como consultantes y tratantes en caso de no haber un intensivista en el turno, personal variable de enfermeras intensivistas y no intensivistas con una relación enfermera: paciente de 1:1 a 1:3 y de enfermera intensivista: paciente de 1:3 a 1:6, tres inhaloterapeutas (en turnos matutino, vespertino y fines de semana), dos residentes rotatorios de neumología (de primer y segundo años) y dos a seis residentes rotatorios por mes de las especialidades de medicina interna, urgencias médicas, anestesiología, medicina crítica y neumología, provenientes del mismo hospital o de otros hospitales. El equipo multidisciplinario de la nueva UCIR es el mismo con que contaba en su antiguo edificio. Las variaciones de personal se dieron antes del traslado al nuevo edificio exclusivamente en el número de intensivistas, con la contratación de un intensivista para el turno vespertino en septiembre de 2015, uno para el turno de noches A en abril de 2016 y uno más para el turno de fines de semana de $8 \mathrm{am}-8 \mathrm{pm}$ en enero de 2017.

\section{Principales diferencias estructurales, de diseño y equipamiento entre las UCIR nueva y antigua}

La nueva UCIR se sitúa en el nuevo edificio para la atención de enfermedades cardiorrespiratorias y vasculares del Hospital General de México, inaugurado en julio de 2017 tras un periodo de construcción de 3 años. Cuenta con un área de $530 \mathrm{~m}^{2}$ con 12 cubículos completamente aislados con puertas de vidrio corredizas y paredes, así como ventanas que dan al exterior. Las tomas de electricidad, aire medicinal, oxígeno y equipamiento están instaladas en la pared posterior a la cabecera del enfermo, y cada cubículo cuenta con lavabo. Dentro del equipamiento, cada cubículo dispone de una cama totalmente automática (Striker, InTouch ${ }^{\circledR}$ ), un sillón reclinable (reposet), un monitor (Philips-IntelliVue ${ }^{\circledR}$ MX800) con opciones de monitorización de gasto cardiaco por método $\mathrm{PiCCO}^{\circledR}$, 
capnografía en tiempo, índice biespectral, variación de presión de pulso entre otros y un ventilador mecánico con capacidad para proporcionar ventilación invasiva y no invasiva (Puritan-Bennett ${ }^{\mathrm{TM}}$ 840). Su diseño cuenta con una entrada con lavabo a manera de transfer para personal de salud y otra para familiares, y una puerta de acceso exclusiva para pacientes en camilla. También dispone de un área de descanso para médicos y una oficina administrativa dentro de la misma unidad. Todo el espacio cuenta con aire acondicionado, inyector y extractor de aire. Además, para el envío y la recepción de muestras biológicas y medicamentos hay un sistema neumático que comunica con las diferentes áreas dentro del edificio.

La UCIR del antiguo edificio estuvo situada en un área adaptada para su uso que data de principios de los años 1900, originalmente destinada para la atención de pacientes tuberculosos. Contaba con siete camas no automáticas separadas por cortinas de material sintético, con un área total de $146 \mathrm{~m}^{2}$, una sola puerta de acceso para pacientes, familiares y personal de salud, que además servía de acceso para ventilación (entrada de aire), un lavabo al ingreso de la unidad, una tarja al centro de la unidad, un baño para el desecho de líquidos orgánicos y un equipamiento consistente en monitores Spacelabs ${ }^{\circledR} \mathrm{mCa}$ re-300 en cada cama con opción de visualización de presiones invasivas y ventiladores mecánicos para ventilación invasiva Viasys ${ }^{\mathrm{TM}}$ Avea $^{\circledR}$ adquiridos en 2009 durante la pandemia de gripe A H1N1. No contaba con área de descanso para médicos ni con área administrativa, y solo tenía dos sillones (reposet) para uso común de pacientes hospitalizados.

El procesamiento de gasometrías y la toma de radiografías portátiles con equipos disponibles in situ son características tanto de la antigua como de la nueva UCIR.

\section{Recolección de datos y análisis estadístico}

Los datos fueron recabados de manera prospectiva de la base de datos DeDUCIR ${ }^{4}$, que cuenta con información demográfica, de gravedad, pronóstico y tiempos de estancia en el hospital y en la UCl, entre otros. Dos médicos de la UCIR son responsables del llenado de la base de datos cada día. Además, la información faltante del egreso hospitalario (fecha y condición de egreso) se recabó directamente del sistema electrónico interno del hospital. Las características de los pacientes se describen utilizando valores absolutos (porcentajes) para las variables dicotómicas y media \pm desviación estándar para las variables continuas. Además del cálculo de la proporción de pacientes vivos observados y esperados al alta hospitalaria, se hizo un análisis del rendimiento clínico y del uso de recursos mediante el método ajustado de Rapoport. La información detallada de la herramienta y su interpretación se describen más adelante en el apartado Métodos. Para el análisis comparativo se usaron datos obtenidos de la antigua UCIR del periodo del 1 de marzo de 2010 al 1 de marzo de $2015^{5}$, y resultados no publicados de rendimiento clínico y de uso de recursos del periodo del 1 de marzo de 2015 al 1 de marzo de 2017. Las características de los pacientes se compararon mediante la prueba de ji al cuadrado de Pearson para las variables dicotómicas y la t de Student para las variables continuas. Un valor de $p<0.05$ indicó significación estadística. En este estudio no aparecen datos de pacientes, su elaboración está planeada dentro de los reportes periódicos de calidad dentro de la institución y no requirió aprobación por los comités institucionales de ética e investigación ni consentimiento informado para su inclusión.

\section{Método revisado de Rapoport-Teres}

El método revisado de Rapoport-Teres ${ }^{1}$ tabula en forma cruzada el rendimiento clínico (índice de rendimiento clínico estandarizado [IRCE]) y el de costo-efectividad (índice de uso de recursos estandarizado [IRURE]) en un gráfico bidimensional. El cálculo de los índices que se detalla a continuación es el mismo que se usó para el análisis de rendimiento clínico y del uso de recursos de la antigua UCIR5:

- IRCE (eje $x$ ): para obtener el índice de rendimiento clínico estandarizado primero se obtiene la diferencia de sobrevida ( $\Delta$ sobrevida) de la población de estudio restando la sobrevida esperada de la sobrevida observada de la siguiente manera:

$$
\begin{aligned}
\Delta \text { sobrevida } & =(\text { sobrevida actual }- \text { sobrevida } \\
& \text { esperada }) \times 100
\end{aligned}
$$

Para expresar este dato en unidades de desviación estándar (estandarizar) de la media de las $\mathrm{UCl}$ de referencia que participaron en el desarrollo del modelo ajustado de Rapoport, a esta diferencia se le extrae la media de la diferencia de sobrevida (0.110) y se divide entre la desviación estándar de la diferencia de sobrevida (4.668) obtenidas del modelo ajustado de Rapoport, y el resultado es el valor del eje $x$ o IRCE. Un valor positivo indica que la diferencia entre la sobrevida observada y la esperada de la $\mathrm{UCl}$ que se 
evalúa es mejor que la del promedio de las $\mathrm{UCl}$ de referencia, mientras que un resultado negativo indica lo contrario.

- IRURE (eje y): para obtener el índice de uso de recursos estandarizado se asigna un valor a los días de estancia en la UCl y a los días pos-UCI para cada paciente. El mayor valor se asigna al primer día de $\mathrm{UCl}$ (4 puntos), a los días subsecuentes en la UCl se les da un valor de 2.5 puntos por día y a los días post-UCl 1 punto por día. El valor promedio de los puntajes obtenidos de todos los pacientes se denomina «días de hospitalización ponderados» (DHP). Al igual que en el análisis de la sobrevida, es necesario disponer de un valor promedio esperado de los días de hospitalización ponderados; para ello, el modelo ajustado de Rapoport ofrece una ecuación derivada de un análisis de regresión que incluye variables independientes que se identificaron como predictoras de la estancia ponderada promedio para la $\mathrm{UCl}$ evaluada:

$\log _{10}(\mathrm{DHP})=1.008+0.753 \times(\%$ muertos en el hospital $)+0.432 \times(\%$ cirugía no programada $)+$

$0.228 \times$ (\% ventilación mecánica en la primera hora de admisión a UCI) $-0.708 \times$ (\% pacientes egresa-

dos a cuidados posagudos fuera del hospital)

Posteriormente se obtiene la diferencia entre los días de hospitalización ponderados esperados y los actuales:

$$
\Delta \mathrm{DHP}=\mathrm{DHP}-\mathrm{DHPe}
$$

Para estandarizar este resultado, al igual que con la sobrevida, a esta diferencia se le sustrae la media de la $\Delta$ DHP $(-0.232)$ y se divide entre la desviación estándar de la $\triangle \mathrm{DHP}$ (3.786), que fueron obtenidos del grupo de UCI del modelo ajustado de Rapoport, y el resultado es el valor del eje y o IRURE. Un resultado positivo indica que se utilizaron menos recursos que los esperados, y un resultado negativo indica lo contrario.

Para su interpretación visual, los valores de IRCE y de IRURE se trasladan a un gráfico de coordenadas de cuatro cuadrantes que permite la comparación de los resultados con los obtenidos por las unidades participantes del proyecto IMPACT en los Estados Unidos de Norteamérica (en desviaciones estándar), y para la comparación de resultados entre periodos distintos en una misma UCl. Las coordenadas en el cuadrante noreste, es decir, los valores de IRCE y de IRURE $>0$, indican mejor sobrevida y menor uso de recursos, mientras que valores negativos de estos índices indican lo contrario.

\section{Resultados}

En el periodo de estudio ingresaron 354 pacientes a la nueva UCIR. Fueron excluidos del análisis los reingresos $(n=27)$, los mayores de 18 años $(n=0)$, los pacientes que permanecían hospitalizados al momento de realizar el análisis $(n=11)$ y aquellos a quienes se limitó o retiró el tratamiento $(n=15)$. En 301 pacientes, la sobrevida hospitalaria observada fue del $63 \%$, mientras que la sobrevida esperada de acuerdo con la escala SAPS-3 fue del $67.7 \%$. Del total de los pacientes, el $9.6 \%$ fallecieron en las primeras 48 horas de ingreso en la UCIR, mientras que el 5.3\% fallecieron en las primeras 24 horas. De acuerdo con el método ajustado de Rapoport, el IRCE fue -1.03 (eje $x$ ) y el IRURE fue 0.05 (eje $y$ ), situando el resultado en coordenadas del cuadrante superior izquierdo del gráfico de Rapoport, dentro de dos desviaciones estándar (Fig. 1). La tabla 1 muestra las características de los pacientes incluidos y su comparación con la población del análisis previo de 2010 a 2015. Además, con los datos disponibles, se muestra la comparación de índices y sus variables incluyendo un periodo adicional (1 de marzo de 2015 a 1 de marzo de 2017) previo a la reubicación de la UCIR. Se observó una diferencia estadísticamente significativa en la sobrevida entre los periodos de análisis de rendimiento comparando el periodo de actividad de la nueva UCIR con el de la antigua en los años 2010 a 2015 (63 vs. $55 \%$; $p=0.01$ ), y una tendencia a una mejor sobrevida comparando el periodo de actividad de la nueva UCIR con el de la antigua en los años 2015 a 2017 (63 vs. 56\%; $p=0.056$ ). Hubo en promedio 25 pacientes atendidos por mes en la nueva UCIR(Tabla 2), frente a 19 pacientes atendidos por mes en los dos periodos previos a su reubicación.

\section{Discusión}

Algunos de los beneficios esperados de medir el rendimiento de una $\mathrm{UCl}$ son poder comparar los resultados con los de otras $\mathrm{UCl}$ consideradas estándar (benchmarking) y hacer comparaciones para una misma unidad en periodos distintos. Además, dado que una UCI puede generar hasta un tercio de los gastos de un hospital, la evaluación del rendimiento y del costo-efectividad es de primordial importancia para la gestión de sus recursos, existiendo para tal efecto numerosas herramientas ${ }^{6-8}$. En 1994, Rapoport et al. ${ }^{9}$ propusieron un modelo bidimensional que tabula en forma cruzada el rendimiento clínico y el uso de 


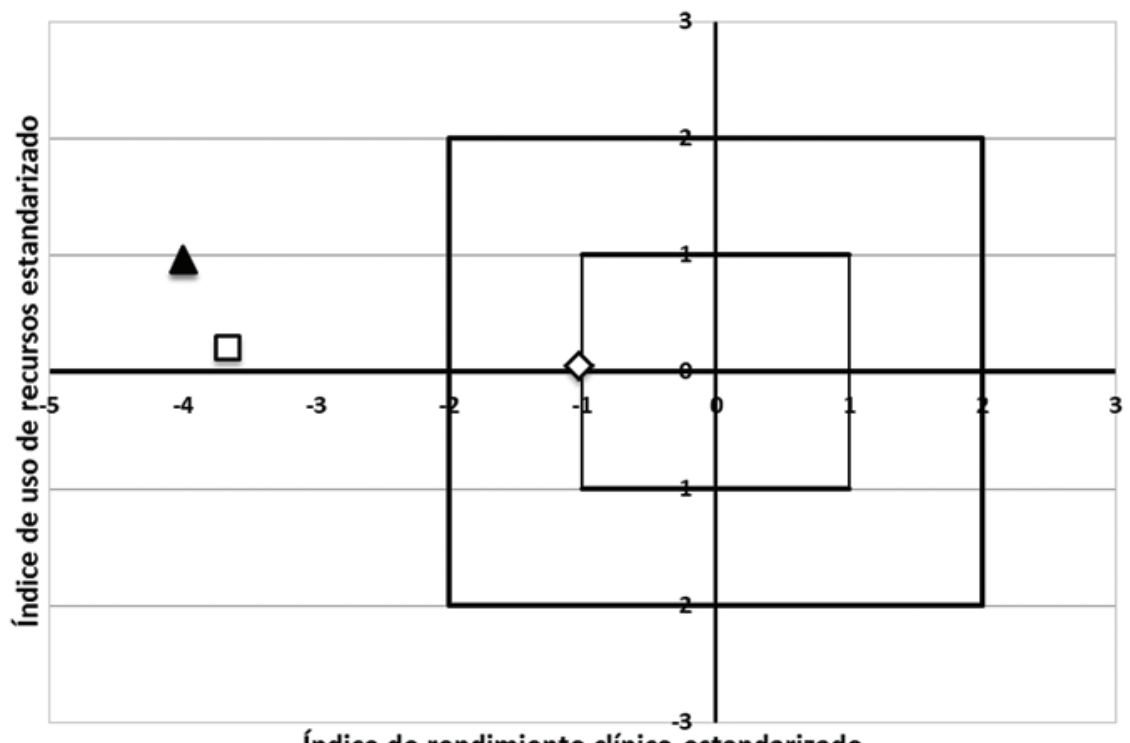

Índice de rendimiento clínico estandarizado

Figura 1. Gráfico del método ajustado de Rapoport para unidades de cuidados intensivos (UCI) graduado en desviaciones estándar (DE). Las coordenadas dentro de $\pm 2 D E$ indican que el rendimiento de una UCl es consistente con el de sus pares, unidades del proyecto IMPACT consideradas "estándar" con las que se compara. El triángulo y el cuadrado indican coordenadas de rendimiento y uso de recursos de la antigua UCIR en los periodos 2010-2015 y 2015-2017, respectivamente; el rombo indica coordenadas de rendimiento y uso de recursos de la nueva UCIR (2017-2018).

recursos usando información relativamente fácil de obtener y un gráfico de coordenadas para la interpretación visual práctica de los resultados. El rendimiento clínico se atribuye a la sobrevida obtenida al alta hospitalaria, mientras que el uso de recursos se basa principalmente en el tiempo de estancia hospitalaria de cada paciente dentro y fuera de la $\mathrm{UCl}$, asignando un valor específico a cada periodo en función del grado de intervenciones que genera (más puntos para el primer día en la UCl). En 2007, el método fue sometido a calibración ${ }^{1}$ y en la actualidad continúa siendo usado por las $\mathrm{UCl}$ del proyecto IMPACT en los Estados Unidos de Norteamérica y por algunas UCI mexicanas como indicador de resultados. En nuestra $\mathrm{UCI}$, la base de datos computada DeDUCIR (que tiene su antecedente en la base de datos local BASU$\mathrm{TI})^{10}$, ha permitido desde su implementación en 2010 realizar este tipo de análisis a la par que otros proyectos ligados a la mejora de la calidad.

Desde el punto de vista organizativo, puede denominarse «recursos" a los componentes de una $\mathrm{UCl}$ destinados a la atención de un paciente en particular, incluyendo no solo los insumos (materiales) sino también el área física, el equipamiento y el personal de salud. Si estos componentes están limitados se espera que el rendimiento clínico sea deficiente ${ }^{2,3,11}$. En el caso particular de la UCIR, antes de su reubicación se observó una tendencia a un mejor rendimiento clínico cuando hubo mayor uso de recursos (días estancia/paciente) $)^{5}$, lo que permitió identificar áreas de oportunidad, como la necesidad de médicos intensivistas para una cobertura asistencial de 24 horas los 7 días de la semana. La labor realizada en los 2 años previos a su reubicación permitió alcanzar un tercio de la plantilla necesaria cubierta por intensivistas certificados; sin embargo, la carencia de intensivistas continúa siendo una de sus principales limitaciones, que de ser subsanada podría llevar a mejores resultados clínicos ${ }^{12}$. El cambio a un área física nueva con diseño y equipamiento adaptados a los estándares actuales permitió a la UCIR equipararse a unidades modélicas en cuestión de estructura y dio paso a un inicio de actividades fresco y motivado por parte de su personal.

Aunque no existe información acerca del desempeño de una UCl durante el inicio de las operaciones con personal de salud ya conocido y con madurez en muchos de sus procesos, se espera que el rendimiento apunte hacia mejores resultados; sin embargo, la inversión en estructura y equipamiento puede llevar a la larga a un gasto todavía mayor en pacientes con un pronóstico malo, que va ligado a la entrega de cuidados avanzados fútiles y a mayor estancia, lo que se ha denominado escalamiento del compromiso en la $\mathrm{UCl}^{13}$. Aunque los pacientes de la nueva UCIR fueron pacientes más graves y hubo un $31 \%$ más de pacientes atendidos por mes en comparación con la antigua UCIR, su sobrevida se aproximó más a la 
Tabla 1. Características de los pacientes en los dos periodos

\begin{tabular}{|c|c|c|c|}
\hline & Antigua UCIR(5) & Nueva UCIR & \multirow[b]{3}{*}{ p } \\
\hline & 2010-2015 & 2017-2018 & \\
\hline & $n=1,136$ & $n=301$ & \\
\hline Edad, años, media \pm DE & $48 \pm 17$ & $50 \pm 15$ & 0.06 \\
\hline $\begin{array}{l}\text { Sexo,masculino/femenino } \\
(\%)\end{array}$ & $544(48)$ & $146(48)$ & 1.0 \\
\hline SAPS $3 \pm$ DE & $51 \pm 19$ & $55 \pm 19$ & 0.001 \\
\hline$S O F A \pm D E$ & $5.2 \pm 4.8$ & $5.0 \pm 4.7$ & 0.5 \\
\hline $\begin{array}{l}\text { Ventilación mecánica } \\
\text { invasiva, } n(\%)\end{array}$ & $704(62)$ & $178(59)$ & 0.34 \\
\hline \multicolumn{4}{|l|}{ Procedencia } \\
\hline $\begin{array}{l}\text { Piso de hospitalización de } \\
\text { neumología }\end{array}$ & $321(28.3)$ & $58(19.3)$ & 0.001 \\
\hline Urgencias & 247 (21.7) & $65(21.6)$ & 0.97 \\
\hline Quirófano de neumología & $241(21.2)$ & 95 (31.6) & $<0.001$ \\
\hline Piso de hospitalización otro & $149(13.1)$ & 49 (16.3) & 0.15 \\
\hline $\begin{array}{l}\text { Quirófano de otros } \\
\text { servicios }\end{array}$ & $116(10.2)$ & $28(9.3)$ & 0.64 \\
\hline Otra UCI & $33(2.9)$ & $2(0.7)$ & 0.02 \\
\hline $\begin{array}{l}\text { Consulta externa de } \\
\text { neumología }\end{array}$ & $19(1.7)$ & $2(0.7)$ & 0.2 \\
\hline Otro hospital & $10(0.9)$ & $2(0.7)$ & 0.7 \\
\hline \multicolumn{4}{|l|}{ Motivo de ingreso en $\mathrm{UCl}$} \\
\hline Falla respiratoria & $523(46.0)$ & $153(50.8)$ & 0.13 \\
\hline Vigilancia posoperatoria & 375 (33.0) & $69(22.9)$ & $<0.001$ \\
\hline Falla cardiovascular & $180(15.8)$ & $60(19.9)$ & 0.08 \\
\hline Falla neurológica & $38(3.3)$ & $5(1.7)$ & 0.14 \\
\hline Falla renal & $13(1.1)$ & $6(2.0)$ & 0.2 \\
\hline Falla metabólica & $5(0.4)$ & $3(1.0)$ & 0.2 \\
\hline Falla hematológica & $1(0.1)$ & $3(1.0)$ & - \\
\hline Falla hepática & $1(0.1)$ & $2(0.7)$ & - \\
\hline Sobrevida hospitalaria (\%) & 55 & 63 & 0.01 \\
\hline
\end{tabular}

DE: desviación estándar; SAPS-3: Simplified Acute Physiology Score 3;

SOFA: Sequential Organ Failure Assessment, UCIR: unidad de cuidados intensivos respiratorios.

esperada por el modelo predictivo SAPS-3, siendo la diferencia estadísticamente significativa en relación al periodo 2010-2015. Otros factores que no se analizan en este reporte pudieron influir de alguna manera en la mejor sobrevida observada en la nueva UCIR. Varios proyectos de mejora de la calidad fueron implementados desde 2010 en la antigua $U \mathrm{CIR}^{4,14,15}$, cuya maduración pudo haber llevado a un impacto cada vez mayor con el pasar de los años, lo que explicaría que en el periodo inmediatamente previo a la reubicación (2015-2017) la diferencia en sobrevida mostrara ya una tendencia a mejores resultados. Por otra parte, la nueva UCIR tuvo un mayor número de pacientes provenientes de quirófano que la antigua, no necesariamente para vigilancia posoperatoria sino con condiciones agudas y presencia de falla en uno o más órganos vitales, en quienes hemos observado
Tabla 2. Variables para el cálculo del IRCE y del IRURE en los diferentes periodos, y resultados por periodo

\begin{tabular}{|c|c|c|c|}
\hline Periodo & $2010-2015^{5}$ & 2015-2017 & 2017-2018* \\
\hline Pacientes incluidos para el cálculo & 1136 & 453 & 301 \\
\hline Sobrevida real & 0,55 & 0,56 & 0,63 \\
\hline Sobrevida esperada por SAPS-3 & 0,74 & 0,73 & 0,67 \\
\hline Diferencia en sobrevida & $-18,56$ & $-16,7$ & $-4,7$ \\
\hline IRCE (eje $x)$ & -4 & $-3,67$ & $-1,03$ \\
\hline Cirugía no programada (\%) & 0,14 & 0,1 & 0,11 \\
\hline Ventilación mecánica (\%) & 0,62 & 0,56 & 0,57 \\
\hline $\begin{array}{l}\text { Alta a cuidados agudos } \\
\text { externos (\%) }\end{array}$ & 0,01 & 0 & 0,01 \\
\hline $\begin{array}{l}\text { Días de hospitalización } \\
\text { ponderados }\end{array}$ & 30,14 & 31,81 & 28,69 \\
\hline $\begin{array}{l}\text { Días de hospitalización } \\
\text { ponderados predichos }\end{array}$ & 33,92 & 32,37 & 28,64 \\
\hline $\begin{array}{l}\text { Diferencia en días de } \\
\text { hospitalización }\end{array}$ & 3,78 & 0,56 & $-0,05$ \\
\hline IRURE (eje y) & 0,94 & 0,21 & 0,05 \\
\hline
\end{tabular}

una asociación con un comportamiento benigno y con mejores resultados en comparación con condiciones médicas graves e inclusive con cirugías de urgencia de otros sistemas orgánicos ${ }^{16,17}$. También, la contaminación ambiental de la $\mathrm{UCl}$ por microorganismos patógenos prevalentes (como por ejemplo Pseudomonas aureuginosa y Acinetobacter baumannii), que desempeña un papel preponderante en el desarrollo de infecciones nosocomiales ${ }^{18}$ y que va ligada a una mayor morbimortalidad, dejó de representar un riesgo en el inicio de las actividades de la nueva UCIR, dado que los pacientes de la antigua fueron tratados hasta su alta o reubicados en otras $\mathrm{UCl}$ dentro del hospital antes de iniciar las labores en el nuevo edificio.

Existen algunas limitaciones, ya comentadas en el análisis de rendimiento clínico y del uso de recursos de la antigua $\mathrm{UCIR}^{5}$, que se repiten para el análisis que se presenta: 1) para el cálculo del IRCE y del IRURE se utilizó la sobrevida esperada de acuerdo con el modelo predictivo de mortalidad SAPS-3 y no con el modelo predictivo MPM- $I_{0}$, con el que la herramienta fue validada; 2 ) al no contar con la proporción de pacientes que requirieron ventilación mecánica en la primera hora de ingreso en la UCIR, se utilizó la proporción total de pacientes en ventilación mecánica para el cálculo del IRURE (este 
aspecto hace que más bien su valor sea infraestimado); y 3) puede ser necesaria una nueva calibración del método de Rapoport para ajustarlo a resultados clínicos y de uso de recursos más contemporáneos en los que influyen los avances tecnológicos y farmacéuticos, y la nueva evidencia científica, entre otros $^{19,20}$. Sin embargo, al ser la comparación entre periodos el motivo principal del análisis, y habiendo usado la misma metodología en los tres periodos que se comparan, pensamos que las modificaciones en el método, aunque limitan la generalización, no impactan de manera importante en el análisis comparativo que se presenta.

\section{Conclusiones}

En su primer año de funcionamiento, la nueva UCIR tuvo mejor rendimiento clínico que la antigua, sin modificación importante en el uso de sus recursos. Existe una asociación entre la mejora de la estructura física y del equipamiento de la UCIR con la progresión a un rendimiento comparable con el de otras $\mathrm{UCI}$ consideradas estándar en el mundo.

\section{Conflicto de intereses}

Los autores declaran no tener conflicto de intereses ni financiamiento relacionados con la preparación de este trabajo. Su realización se apega a los requisitos éticos de la institución donde fue elaborado.

\section{Responsabilidades éticas}

Protección de personas y animales. Los autores declaran que para esta investigación no se han realizado experimentos en seres humanos ni en animales.

Confidencialidad de los datos. Los autores declaran que en este artículo no aparecen datos de pacientes.

Derecho a la privacidad y consentimiento informado. Los autores han obtenido el consentimiento informado de los pacientes y/o sujetos referidos en el artículo. Este documento obra en poder del autor de correspondencia.

\section{Bibliografía}

1. Nathanson BH, Higgins TL, Teres D, Copes WS, Kramer A, Stark M $A$ revised method to assess intensive care unit clinical performance and resource utilization. Crit Care Med. 2007;35:1853-62.

2. Schultz MJ, Dunser MW, Dondorp AM, Adhikari NK, Iyer S, Kwizera A, et al. Current challenges in the management of sepsis in ICUs in resource-poor settings and suggestions for the future. Intensive Care Med. 2017;43:612-24.

3. Geiling J, Burkle FM Jr, Amundson D, Dominguez-Cherit G, Gomersall CD, Lim ML, et al. Resource-poor settings: infrastructure and capacity building: care of the critically ill and injured during pandemics and disasters: CHEST consensus statement. Chest. 2014;146(4 Suppl):e156S-67S.

4. Álvarez-Maldonado $P$, Cueto-Robledo G, Cerón-Díaz U, Pérez-Rosales A Navarro-Reynoso F, Cicero-Sabido R. Indicadores de calidad en una unidad de cuidados intensivos respiratorios. Análisis inicial de la base de datos DEDUCIR. Med Intensiva. 2012;36:518-20.

5. Álvarez-Maldonado P, Pérez-Rosales A, Cueto-Robledo G, Cerón-Díaz U, Núñez-Pérez-Redondo $C$, Navarro-Reynoso $F$, et al. Clinical performan$c e$ and resource use of a respiratory intensive care unit. Analysis of five years of clinical activity. Rev Med Hosp Gen Mex. 2016;79:144-9.

6. Elguea Echavarría PA, Cerón Díaz UW, Esponda Prado JG, Cabrera Jardines R. Calidad y costo-efectividad en la atención del paciente crítico. Rev Asoc Mex Med Crit Ter Int. 2012;26:42-50.

7. Villagómez Ortiz AJ. Costo efectividad de la atención en cuidados intensivos. Rev Asoc Mex Med Crit Ter Int. 2012;4:192-3.

8. Timmers TK, Verhofstad MH, Moons KG, Leenen LP. Intensive care performance: how should we monitor performance in the future? World J Crit Care Med. 2014;3:74-9.

9. Rapoport J, Teres D, Lemeshow S, Gehlbach S. A method for assessing the clinical performance and cost-effectiveness of intensive care units: a multicenter inception cohort study. Crit Care Med. 1994;22:1385-91.

10. Cerón U, Sierra A, Martínez R, Vázquez JP. Base de datos para el control de calidad y utilización de recursos en la unidad de terapia intensiva. Rev Mex Med Crit Ter Int. 1996;10:105-201.

11. Dat VQ, Long NT, Giang KB, Diep PB, Giang TH, Diaz JV. Healthcare infrastructure capacity to respond to severe acute respiratory infection (SARI) and sepsis in Vietnam: a low-middle income country. J Crit Care. 2017;42:109-15

12. Neuraz A, Guérin C, Payet $C$, Polazzi $S$, Aubrun F, Dailler F, et al. Patient mortality is associated with staff resources and workload in the ICU: a multicenter observational study. Crit Care Med. 2015;43:1587-94.

13. Braxton CC, Robinson CN, Awad SS. Escalation of commitment in the surgical ICU. Crit Care Med. 2017;45:e433-e436.

14. Alvarez-Maldonado P, Cueto-Robledo G, Cicero-Sabido R. Changes observed in three quality indicators after the implementation of improvement strategies in the respiratory-ICU. Med Intensiva. 2015;39:142-8.

15. Álvarez-Maldonado $P$, Reding-Bernal A, Hernández-Solís A, Cicero-Sabido R. Impact of strategic planning, organizational culture imprint and care bundles to reduce adverse events in the ICU. Int J Qual Health Care. 2019;31:480-4.

16. Fernández MA, de la Escosura G, Cicero R. Valoración de APACHE II. Experiencia en dos grupos con patología neumológica. Rev Inst Nal Enf Resp Mex. 1996;3:187-93.

17. Álvarez-Maldonado $P$, Pérez-Rosales $A$, Núñez-Pérez Redondo $C, N a-$ varro-Reynoso F, Cicero-Sabido R. Cirugía de urgencia torácica versus no torácica. Resultados en una unidad de cuidados intensivos respiratorios. Rev Asoc Mex Med Crit Ter Int. 2015;19:133-7.

18. Russotto V, Cortegiani A, Fasciana T, lozzo P, Raineri SM, Gregoretti C, et al. What healthcare workers should know about environmental bacterial contamination in the intensive care unit. Biomed Res Int. 2017;2017:6905450.

19. Abascal Caloca C, Cerón Díaz UW, Vázquez Mathieu JP, Sierra Unzueta A. Análisis de costo-efectividad por el método de Rapoport en un año de actividad asistencial en una unidad de terapia intensiva polivalente privada en México. Rev Asoc Mex Med Crit Ter Int. 2006;1:5-11.

20. Cerón Díaz UW, Abascal C, Sierra Unzueta A. Comparación del rendimiento clínico y costo-efectividad en tres periodos durante nueve años de actividad asistencial de una unidad de terapia intensiva. Rev Asoc Mex Med Crit Ter Int. 2006;20:126-35. 